\title{
G04genome: A Prokaryotic Phylogeny Based on Genome Organization
}

\author{
Rainer Merkl · Arnim Wiezer
}

Received: 27 June 2008/Accepted: 3 April 2009/Published online: 13 May 2009

(C) Springer Science+Business Media, LLC 2009

\begin{abstract}
Determining the phylogeny of closely related prokaryotes may fail in an analysis of rRNA or a small set of sequences. Whole-genome phylogeny utilizes the maximally available sample space. For a precise determination of genome similarity, two aspects have to be considered when developing an algorithm of whole-genome phylogeny: (1) gene order conservation is a more precise signal than gene content; and (2) when using sequence similarity, failures in identifying orthologues or the in situ replacement of genes via horizontal gene transfer may give misleading results. GO4genome is a new paradigm, which is based on a detailed analysis of gene function and the location of the respective genes. For characterization of genes, the algorithm uses gene ontology enabling a comparison of function independent of evolutionary relationship. After the identification of locally optimal series of gene functions, their length distribution is utilized to compute a phylogenetic distance. The outcome is a classification of genomes based on metabolic capabilities and their organization. Thus, the impact of effects on genome organization that are not covered by methods of molecular phylogeny can be studied. Genomes of strains belonging to Escherichia coli, Shigella, Streptococcus, Methanosarcina,
\end{abstract}

Electronic supplementary material The online version of this article (doi:10.1007/s00239-009-9233-6) contains supplementary material, which is available to authorized users.

\section{R. Merkl ( ()}

Institut für Biophysik und Physikalische Biochemie, Universität Regensburg, 93040 Regensburg, Germany

e-mail: rainer.merkl@biologie.uni-regensburg.de

A. Wiezer

Göttingen Genomics Laboratory, Grisebachstrasse 8,

37077 Göttingen, Germany and Yersinia were analyzed. Differences from the findings of classical methods are discussed.

Keywords Whole-genome phylogeny ·

Bacterial genomes · Computational molecular biology . Prokaryotic phylogenomics · Gene ontology

\section{Introduction}

The classical approach of phylogenetic categorization relies on the analysis of rRNA sequences as introduced by C. Woese (Woese and Fox 1977). However, if the sequences are too similar, it is not possible to determine an evolutionary relationship precisely. This is frequently the case when studying closely related species. For these applications, genome-based phylogenies are superior: the number of mutations separating species will increase with the number of genes analyzed. In addition, methods exploiting a larger number of genes are less affected by horizontal gene transfer (HGT), variable mutation rates, or misalignments (Snel et al. 1999; Fitz-Gibbon and House 1999). For these reasons, phylogenomic methods that use a large set of sequences have become the de facto standard for reconstructing phylogenies (Ciccarelli et al. 2006; Daubin et al. 2002), especially for closely related species (Oshima and Nishida 2007). The algorithms for genomebased phylogeny can be grouped according to their concepts. There are methods that compare genomic DNA sequences on the whole and methods that evaluate gene content or gene order. So far, sequence methods have been used most frequently. In this case, genomes are compared pairwise at the DNA level (Kurtz et al. 2004; Darling et al. 2004). These methods can be extended to construct phylogenetic trees (Henz et al. 2005). 
The classical gene content methods are, compared to the above, more complex and require several steps. First, orthology of genes has to be determined. Then the occurrence or absence of genes has to be evaluated and used to infer a phylogenetic tree (see Snel et al. 1999; Bapteste et al. 2004; Lin et al. 2009). Alternatively, the sequences of a small set of genes (Ciccarelli et al. 2006; Konstantinidis et al. 2006) or of a core genome can be concatenated prior to phylogenetic analysis. It has been shown that 20 genes were sufficient for a phylogenetic analysis of eight yeast taxa (Rokas et al. 2003). However, these approaches have been criticized: For a set of $205 \gamma$-proteobacterial core genes it has been demonstrated that their history is unknown in many cases and that these genes rarely favor one phylogenetic tree (Susko et al. 2006; Bapteste et al. 2008).

In addition to content, gene order can be utilized to compare genomes. Quite sophisticated theoretical concepts have been developed for the assessment of genomic rearrangements (Sankoff 1992; Hannenhalli et al. 1995) and implemented for their analysis (Tesler 2002; Dalevi and Eriksen 2008). However, to the best of our knowledge, the only approach used so far for comparison of complete microbial genomes is the SHOT server, which exploits the occurrence of gene pairs (Korbel et al. 2002).

One goal of whole-genome comparison is the determination of the true evolutionary distance, i.e., the actual number of mutational events separating two genomes. Unfortunately, this distance cannot be inferred. As a substitute, an edit distance may be computed. The edit distance is the minimal number of evolutionary events selected from a predefined set of operations that transform one genome into the other one. However, even the computation of an edit distance is known to be NP-hard for genomes with unequal content (Xin et al. 2005). Definitely, this time complexity is a severe hindrance for using exact methods to analyze native genomes. Exact methods suffer from a second restriction: these algorithms consider the problem of determining the identity of genes as being solved. As a prerequisite, each gene has to be labeled with a number indicating the orthology class it belongs to. If sequence comparison is the basis to identify orthologues, it may fail: due to gene duplication, several paralogues may exist. In these cases, sequence similarity is no clear indicator of evolutionary relationship.

In addition, it has been proved plausible that HGT is a major force shaping the content of microbial genomes (see, e.g., Ochman et al. 2000 and references therein). Lawrence and Ochman (1997) proposed that at least $15 \%$ of the $E$. coli genome is atypical and may have arisen by recent transfer events. It has been concluded that $25 \%$ of the Thermotoga maritima genes are more closely related to archeal genes and may signal gene transfer between these lineages (Nelson et al. 1999). For M. mazei it has been postulated that up to $30 \%$ of the genome may have been acquired via HGT (Deppenmeier et al. 2002). However, the extent and the long-term impact of HGT on individual genomes are still a matter of debate (see, e.g., Kurland et al. 2003).

It has been shown that genes may be replaced in situ with nonorthologous ones (Omelchenko et al. 2003). In these cases, the function of the gene product remains the same, which may not be detectable when comparing sequences. How such an event must be assessed with respect to phylogenetic analysis is debatable.

Due to these arguments, we introduce and apply a new paradigm for genome comparison: we consider genomes as a set of gene series implementing certain functions. The algorithm, which we named GO4genome, is based on the pairwise comparison of gene function and gene order. For assessment of function, it does not consider homology, i.e., evolutionary relationship. Instead, the algorithm utilizes gene ontology. For comparison of gene order, we introduce a heuristic approach. The algorithm identifies the longest series of genes possessing the most similar function. The number and length of these series are then used to compute pairwise genomic distances, which are the basis for phylogenetic inference. Thus, GO4genome comprises additional events like genomic rearrangements for comparison of genomes, which are beyond those exploited by methods of molecular phylogeny. We demonstrate for several groups of microbes that the inferred phylogenetic relationship is, in most cases, in agreement with the outcome of classical methods. A novel grouping of species was observed, e.g., in rapidly evolving genomes like those of Yersinia pestis strains or in Shigella.

\section{Materials and Methods}

\section{Datasets}

For all analyses, entries downloaded from the Genome Reviews database (http://www.ebi.ac.uk/GenomeReviews/) were utilized; it provides comprehensively annotated data, including gene ontology (GO) terms. The following datasets were used (accession numbers in parentheses).

\section{Escherichia coli Dataset}

The E. coli dataset was comprised of E. coli EDL933 (AE005174_GR.gbk), E. coli K-12 (U00096_GR.gbk), E. coli Sakai (BA000007_GR.gbk), E. coli UTI89 (CP000 243_GR.gbk), E. coli O1K1 / APEC (CP000468_GR.gbk), E. coli CFT073 (AE014075_GR.gbk), E. coli 536 (CP00 0247_GR.gbk), S. boydii strain Sb227(CP000036_GR. 
gbk), S. dysenteriae strain Sd197 (CP000034_GR.gbk), S. flexneri ATCC 700930 (AE014073_GR.gbk), S. flexneri strain 301 (AE005674_GR.gbk), S. flexneri strain 8401 (CP000266_GR.gbk), S. sonnei strain Ss046 (CP000038_ GR.gbk), S. typhimurium LT2 (AE006468_GR.gbk), B. aphidicola (BA000003_GR.gbk), R. conorii (AE006914_ GR.gbk), R. prowazekii (AJ235269_GR.gbk), Y. pestis Antiqua (CP000308_GR.gb), and A. pernix (BA000002_ GR.gbk).

\section{Streptococcus Dataset}

The Streptococcus dataset included $S$. agalactiae III (AL732656_GR.gbk), S. agalactiae Ia (CP000114_GR. gbk), S. agalactiae V (AE009948_GR.gbk), S. mutans (AE014133_GR.gbk), S. pneumoniae NCTC7466 (CP000 410_GR.gbk), S. pneumoniae R6 (AE007317_GR.gbk), S. pneumoniae TIGR4 (AE005672_GR.gbk), S. pyogenes M12 MGAS2096 (CP000261_GR.gbk), S. pyogenes M12 MGAS9429 (CP000259_GR.gbk), S. pyogenes M2 MGAS 10270 (CP000260_GR.gbk), S. pyogenes M4 MGAS10750 (CP000262_GR.gbk), S. pyogenes M5 (AM295007_GR. gbk), S. pyogenes M1 MGAS5005 (CP000017_GR.gbk), S. pyogenes M1 ATCC700294 (AE004092_GR.gbk), S. pyogenes M18 MGAS8232 (AE009949_GR.gbk), S. pyogenes M28 MGAS6180 (CP000056_GR.gbk), S. pyogenes M3 MGAS315 (AE014074_GR.gbk), S. pyogenes M3 SSI_1 (BA000034_GR.gbk), S. pyogenes M6 MGAS10394 (CP000003_GR.gbk), S. sanguinis (CP000387_GR.gbk), S. suis 05ZYH33 (CP000407_GR.gbk), S. suis 98HAH33 (CP000408_GR.gbk), S. thermophilus LMG18311 (CP000 023_GR.gbk), S. thermophilus LMD9 (CP000419_GR. gbk), and S. thermophilus CNRZ1066 (CP000024_GR.gbk).

\section{Methanosarcina Dataset}

The Methanosarcina dataset comprised M. mazei (AE00 8384_GR.gbk), M. barkeri (CP000099_GR.gbk), M. acetivorans (AE010299_GR.gbk), M. thermophila (CP000477_ GR.gbk), M. hungatei (CP000254_GR.gbk), M. marisnigri (CP000562_GR.gbk), M. labreanum (CP000559_GR. gbk), T. acidophilum (AL139299_GR.gbk), T. volcanium (BA000011_GR.gbk), P. horikoshii (BA000001_GR.gbk), P. abyssi (AL096836_GR.gbk), and P. furiosus (AE0099 50_GR.gbk).

\section{Yersinia Dataset}

The Yersinia dataset included Y. enterocolitica (AM2864 15_GR.gbk), $Y$. pestis Antiqua (CP000308_GR.gb), $Y$. pestis Nepal516 (CP000305_GR.gbk), Y. pestis Mediaevalis 91001 (AE017042_GR.gbk), Y. pestis Orientalis CO92 (AL590842_GR.gbk), Y. pestis Pestoides F (CP0006
68_GR.gbk), Y. pestis Mediaevalis KIM5 (AE009952_GR. gbk), and Y. pseudotuberculosis (BX936398_GR.gbk).

Computing funSim Values for Genomes

For each genome, a file was created containing, in multiple FASTA format, GO terms for each gene separated according to the three GO categories "cellular component," "biological process" (BP), and "molecular function" (MF). The program funSim (Schlicker et al. 2006) version 1.0 was used to compare genomes pairwise. The score was deduced from the categories BP and MF. The output of funSim is a distance matrix storing for each pair of genes $a_{i}, b_{j}$ the value $\operatorname{fun} \operatorname{Sim}\left(a_{i}, b_{j}\right)$. For each pair of genomes $G k, G l$ belonging to a dataset under study, such a matrix (GkGl_matrix) was computed.

Computing Phylogenetic Distance Matrices by Means of GO4genome

According to the dataset $G 1 \ldots G n$ to be analyzed, GO4genome reads the respective $G k G l \_m a t r i c e s$ and computes for each pair of genomes a Dist $_{G O}$ value as described under Results and according to Formula (6). The set of Dist $_{G O}$ values is written to a file in Nexus format (Maddison et al. 1997). The source code for the generation of Nexus-formatted distance matrices and the yersiniae dataset can be downloaded from http://www-bioinf.uniregensburg.de.

\section{Creating Neighbor Nets}

For the visualization of results, we utilized the program SplitsTree4 (version 4.8) (Huson and Bryant 2006). The output of GO4genome was fed into SplitsTree4. Neighbor nets were created by using default parameters.

\section{Results}

Toward a Novel Algorithm of Genome Comparison Based on Gene Ontology Annotations

As it was our aim to develop a method for the comparison of genomes which exploits encoded function, we first focused on an adequate scoring scheme. So far, gene content methods have been based exclusively on the concept of homology. For assessment of this approach, the following characteristics have to be considered. (1) This categorization of genes (gene products) is a binary one. Definitely, a scoring scheme with finer granularity supports a more precise comparison of genomic content, which is less error prone also. (2) The 
classification may fail on paralogues. It was shown that gene duplication is an important factor in genome evolution (Snel et al. 2002). (3) This classification is based on a common evolution of respective genes. In cases where a nonorthologous in situ replacement of a gene via HGT preserves function, the analysis of homology will report disparate genome content.

With the advent of gene ontology (GO), this binary classification scheme can easily be replaced by a continuous one. GO is a standardized vocabulary permitting a coherent annotation of gene products. It is now common to supply genes and gene products with a set of GO terms annotating, e.g., function or their involvement in biological processes. Recently, methods for comparing sets of GO terms have been introduced (Del Pozo et al. 2008; Schlicker et al. 2006). The latter method relies on two similarity measures; one, named funSim, can be used to characterize the functional similarity of gene products. It has been shown that this identification of functionally related proteins is independent of their evolutionary relationship (Schlicker et al. 2006). The outcome of funSim is, for each pair of genes $a_{i}, b_{j}$, a score $0.0 \leq \operatorname{fun\operatorname {Sim}}\left(a_{i}\right.$, $\left.b_{j}\right)<1.0$. For the following, we assume that genome $G 1$ consists of $n$ genes $a_{1}, a_{2}, \ldots, a_{n}$, and genome $G 2$ of $m$ genes $b_{1}, b_{2}, \ldots, b_{m}$, being annotated with GO terms. In addition, it is assumed that $m \leq n$, which can always be ascertained by changing indices, if necessary. A matrix $G O_{-} S\left[a_{1} \ldots a_{n}\right]$ $\left[b_{1} \ldots b_{m}\right]$ can be computed, which harbors all funSim $\left(a_{i}, b_{j}\right)$ values. In analogy to classical scoring matrices, $G O \_S$ constitutes a basis for the comparison of $G 1$ and $G 2$ in gene function.

For analyses described below, we utilized the annotations deposited in the Genome Reviews database of the EBI (see "Materials and Methods"), which provides comprehensively annotated genomes. A typical example is Escherichia coli K-12 (accession number U00096_GR.gbk). This dataset contained 4277 genes; 3496 have been annotated with GO terms. Of the remaining 781 genes, 462 have been described as "hypothetical" or "uncharacterized"; most of the other annotations are nonspecific. Therefore, one can assume that the largest fraction of shared genes has been provided with GO terms, putting an analysis on a sound basis.

As explained above, current algorithms for genome comparison are based on a binary classification of genes. Additionally, those classical algorithms for sequence comparison (Smith and Waterman 1981) which can utilize a scoring system cannot deal with inversions. However, this kind of genetic rearrangement occurs quite frequently, even in closely related genomes (Hughes 2000; Belda et al. 2005). Therefore, we propose a novel method which rests on the identification of high-scoring segments as BLAST does (Altschul et al. 1990).

Identifying Gene Series of Maximal Length with the Most Similar Function

An approximation for computing an edit distance is the construction of a cover (Swenson et al. 2008). A cover consists of a series of genes that exist in both genome $G 1$ and genome $G 2$. A cover is said to be optimal if it corresponds to the minimal number of edit operations needed to transform $G 1$ into $G 2$. However, the computation of an optimal cover is NP-hard (see Swenson et al. 2008). Therefore, a minimal cover that consists of the smallest number of series is used as a surrogate (Swenson et al. 2008).

Here we propose an algorithm that identifies a functionally minimal cover for the genomes $G 1$ and $G 2$. The algorithm utilizes the matrix $G O \_S\left[a_{1} \ldots a_{n}\right]\left[b_{1} \ldots b_{m}\right]$. $G O \_S$ values were used to identify high scoring 3-tuples of genes (called HS3Ts or A_HS3Ts). We selected tuples of length 3 , as these are the shortest $n$-mers allowing the identification of local optima. HS3Ts were determined according to the following rules and stored in a matrix $T G$ of size $n \times m$ :

$H S 3 T[i, j]= \begin{cases}1 & \text { if } \operatorname{diag}(i, j)=\text { true } \\ 0 & \text { if } \operatorname{diag}(i, j)=\text { false }\end{cases}$

The value of $\operatorname{diag}(i, j)$ originated from the following expression (compare Supplementary Fig. S1, Panel A):

$$
\begin{aligned}
& \left(G O \_S\left[a_{i}, b_{j}\right] \geq G O \_S\left[a_{i}, b_{j+1}\right]\right) \wedge\left(G O \_S\left[a_{i}, b_{j}\right]\right. \\
& \left.\geq G O \_S\left[a_{i}, b_{j-1}\right]\right) \\
& \wedge\left(G O \_S\left[a_{i}, b_{j}\right] \geq G O \_S\left[a_{i-1}, b_{j}\right]\right) \wedge\left(G O \_S\left[a_{i}, b_{j}\right]\right. \\
& \left.\geq G O \_S\left[a_{i+1}, b_{j}\right]\right) \\
& \wedge\left(G O \_S\left[a_{i-1}, b_{j-1}\right] \geq G O \_S\left[a_{i-1}, b_{j}\right]\right) \wedge\left(G O \_S\left[a_{i-1}, b_{j-1}\right]\right. \\
& \left.\geq G O \_S\left[a_{i}, b_{j-1}\right]\right) \\
& \wedge\left(G O \_S\left[a_{i+1}, b_{j+1}\right] \geq G O \_S\left[a_{i+1}, b_{j}\right]\right) \wedge\left(G O \_S\left[a_{i+1}, b_{j+1}\right]\right. \\
& \left.\geq G O \_S\left[a_{i}, b_{j+1}\right]\right)
\end{aligned}
$$

For $H S 3 T[i, j]=1$, three neighboring elements of $T G$ were set to 1 according to $T G[i, j]=T G[i+1, j+1]=T G$ $[i-1, j-1]=1$.

Analogously, stretches indicating genomic inversions were identified:

$A \_H S 3 T[i, j]= \begin{cases}1 & \text { if } A \_ \text {diag }(i, j)=\text { true } \\ 0 & \text { if } A \_ \text {diag }(i, j)=\text { false }\end{cases}$ 
A_diag $(i, j)$ is the result of the following term (compare Supplementary Fig. S1, Panel B):

$$
\begin{aligned}
& \left(G O \_S\left[a_{i}, b_{j}\right] \geq G O \_S\left[a_{i}, b_{j+1}\right]\right) \wedge\left(G O \_S\left[a_{i}, b_{j}\right]\right. \\
& \left.\geq G O \_S\left[a_{i}, b_{j-1}\right]\right) \\
& \wedge\left(G O \_S\left[a_{i}, b_{j}\right] \geq G O \_S\left[a_{i-1}, b_{j}\right]\right) \wedge\left(G O \_S\left[a_{i}, b_{j}\right]\right. \\
& \left.\geq G O \_S\left[a_{i+1}, b_{j}\right]\right) \\
& \wedge\left(G O \_S\left[a_{i+1}, b_{j-1}\right] \geq G O \_S\left[a_{i+1}, b_{j}\right]\right) \wedge\left(G O \_S\left[a_{i+1}, b_{j-1}\right]\right. \\
& \left.\geq G O \_S\left[a_{i}, b_{j-1}\right]\right) \\
& \wedge\left(G O \_S\left[a_{i-1}, b_{j+1}\right] \geq G O \_S\left[a_{i}, b_{j+1}\right]\right) \wedge\left(G O \_S\left[a_{i-1}, b_{j+1}\right]\right. \\
& \left.\geq G O \_S\left[a_{i-1}, b_{j}\right]\right)
\end{aligned}
$$

If $A \_H S 3 T[i, j]$ was 1 , the content of $T G$ was altered according to $T G[i, j]=T G[i-1, j+1]=T G[i+1, j-1]$ $=1$.

It is reasonable to prevent the further assessment of a pair of genes $a_{i}, b_{j}$ that do not have similar function. Therefore, we introduced a lower limit GO_cut_off when filling GO_S. Besides unrelated function, low funSim values might originate from inadequate annotation quality, from inconsistencies in the ontology, or from errors in the funSim implementation. To assess funSim values, we utilized GO4genome to compare all genes $a_{j}$ of those 19 genomes $G i$ constituting the $E$. coli dataset (see below) with themselves and determined the distribution of fun$\operatorname{Sim}_{G i G i}\left(a_{j}, a_{j}\right)$ values. Altogether 48,746 gene pairs were analyzed; less than $5 \%$ had funSim values $<0.59$, and more than $90 \%$ a funSim value $\geq 0.87$. Therefore, we selected $G O \_c u t \_o f f=0.59$. These results also confirmed that the annotations as deposited in the Genome Reviews database as well as the implementation of funSim are of high quality. We confirmed that the outcome of GO4genome does not depend critically on this parameter. Supplementary Fig. S2 allows comparison of analyses of the E. coli dataset based on $G O \_c u t \_o f f$ values of $0.59,0.68$, and 0.75 .

If HS3Ts overlapped, longer diagonal elements $\operatorname{diag}\left(a_{i}, b_{j}, a_{k}, b_{l}\right)$ resulted, extending from position $i, j$ to position $k, l$. The same could be the case for $A \_H S 3 T s$. All diagonal elements occurring in $T G$ were sorted according to their length and stored in a list, DIAG_LIST. In the next step, an optimal set of diagonal elements was selected in order to label genes $b_{1}$ to $b_{m}$. Starting with the element $\operatorname{diag}\left(a_{i}, b_{j}, a_{k}, b_{l}\right)$ of maximal length, genes $b_{j}$ to $b_{l}$ were labeled. In addition, all elements of any $\operatorname{diag}_{m}$ belonging to the corresponding intervals $a_{i} \ldots a_{k}$ or $b_{j} \ldots b_{l}$ were removed. Entries in DIAG_LIST were processed until all genes $b_{1}$ to $b_{m}$ were labeled or until DIAG_LIST was empty. The result of this process is a set of diagonal elements (a functionally minimal cover) $S_{-} D I A G$ that contains all genes $b_{j}$ of $G 2$ possessing a significant functional similarity to genes of $G 1$. Please note that, due to this filter, gene pairs $a_{i}, b_{j}$ possessing the highest funSim values are not necessarily elements of $S \_D I A G$. This set may contain crosswisearranged elements, which could be separated by gaps of arbitrary lengths (compare Supplementary Fig. S3). Figure 1 shows that the set of genes constituting $S_{-} D I A G$ and those sequences aligned by MUMmer or generated by a pairwise BLAST analysis overlap to a great extent. Using the above results, a distance Dist $_{G O}$ for $G 1$ and $G 2$ was calculated according to the following formulae:

$\operatorname{sim}_{G O}\left(\operatorname{diag}_{k}\right)=\sum_{a_{i}, b_{j} \in \operatorname{diag}_{k}} \operatorname{funSim}\left(a_{i}, b_{j}\right)$

$$
\begin{aligned}
& \operatorname{Dist}_{G O}(G 1, G 2) \\
& =-\log \left(\sum_{\text {diag }_{k} \in S \_D I A G}\left(\frac{\operatorname{sim}_{G O}\left(\operatorname{diag}_{k}\right)}{\text { weighted_gsize }(G 1, G 2)}\right)^{\lambda}\right)
\end{aligned}
$$

weighted_gsize $(G 1, G 2)=\frac{\sqrt{2} \cdot \operatorname{size}(G 1) \cdot \operatorname{size}(G 2)}{\sqrt{\operatorname{size}(G 1)^{2}+\operatorname{size}(G 2)^{2}}}$

$\operatorname{sim}_{G O}\left(\operatorname{diag}_{k}\right)$ is the sum of all funSim values for those gene pairs constituting one element $k \in S_{-} D I A G$. If two neighboring elements $\operatorname{diag}_{k}$, $\operatorname{diag}_{l}$ occupied the same diagonal line, $\operatorname{sim}_{G O}$-values were merged (see Supplementary Fig. S3). For the computation of a distance, $\operatorname{sim}_{G O}\left(\operatorname{diag}_{k}\right)$ values were divided by the weighted average genome size, weighted_gsize(G1,G2), in analogy to Korbel et al. (2002). For Formula (6) we propose to use a $\lambda$ which is $>1.0$. In this case, any combination of two or more normalized $\operatorname{sim}_{G O}\left(\operatorname{diag}_{k}\right)$ values (indicating rearrangements) will sum up to a value which is $<1.0$. The comparison of trees deduced for the E. coli dataset (data not shown) proved that $\lambda=1.05$ is appropriate.

The evolutionary distance $\operatorname{Dist}_{G O}(G 1, G 2)$ was deduced from the estimated similarity by applying the negative logarithm, as proposed by Korbel et al. (2002). Please note that short fragments contribute only marginally to the distance value; see Formula (6). Therefore, we did not consider elements consisting of fewer than three gene pairs; compare Formulae (2) and (4). For the E. coli dataset (see below), the number of elements making up individual sets $S \_D I A G$ varied between 1 and 208.

For a set of genomes $\mathrm{G} 1 \ldots \mathrm{G} n$, the outcome of all pairwise comparisons $\mathrm{G} i, \mathrm{G} j$ is a distance matrix of size $n \times n$. A frequently used method for the construction of a tree is some variant of a neighbor joining algorithm (Saitou and Nei 1987). The resulting tree will be free of ambiguities, if the distance matrix is additive. However, for the general case, we did not expect additive matrices when comparing several genomes via GO4genome. If conflicting signals (i.e., distances) exist, a neighbor net can be used for indication. We utilized the version implemented with SplitsTree4 (Huson and Bryant 2006). 

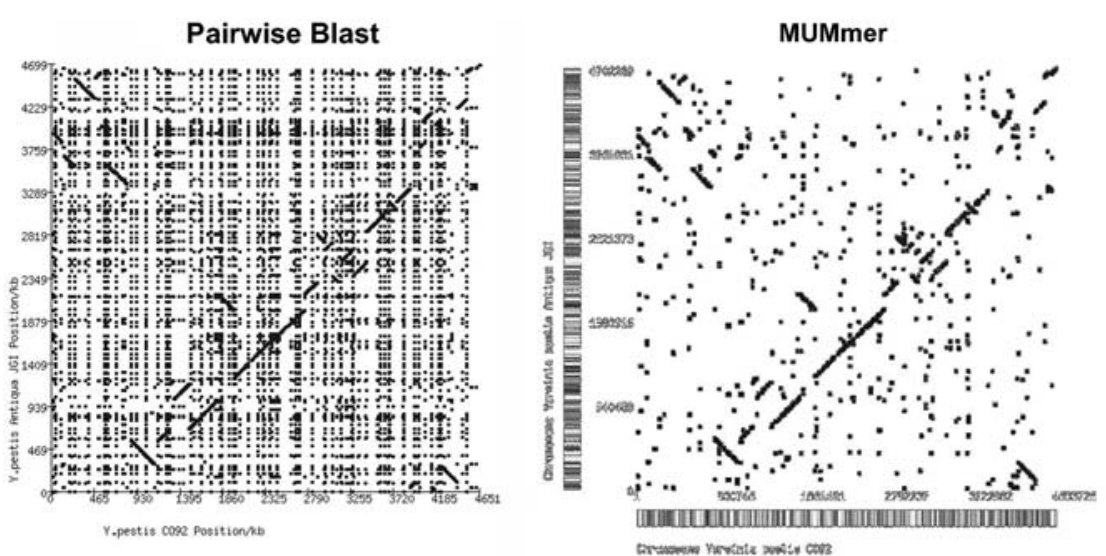

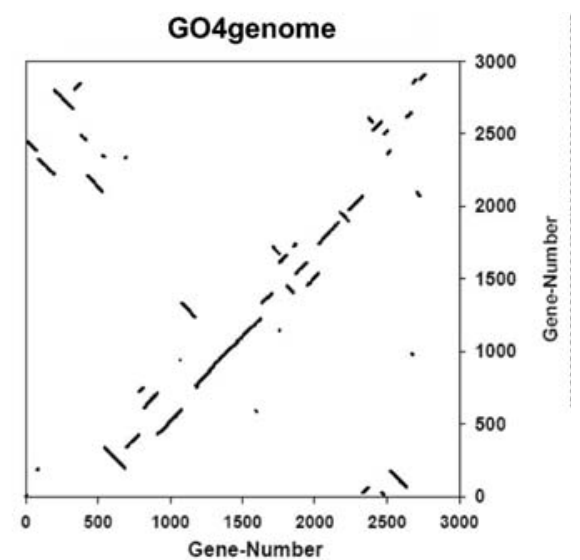

Due to rigorous filtering, which is due to the specific selection of diagonal elements, these duplicates do not occur in the GO4genome plot. For determination of dot-plots based on MUMmer or BLAST hits, we utilized the tools offered at the Comparative Tools page of the JCVI (http://cmr.jcvi.org/). The genome of $Y$. pestis CO-92 is plotted on the abscissa

identity tree (see Konstantinidis et al. 2006). All Shigella genomes were grouped together; the three $S$. flexneri strains cluster in one distinct group. S. boydii, S. dysenteriae, and $S$. sonnei constitute a second cluster. A phylogenetic analysis of shigellae, based on smaller sets of gene sequences, resulted in inconsistent phylogenies (see Yang et al. 2007); see the "Discussion".

Buchnera, Y. pestis, Rickettsia, and A. pernix were more distant from the other species. The positioning of Buchnera is a specific challenge, as the genome of this endosymbiont has undergone massive genome reduction since the divergence from a free-living $\gamma$-proteobacterial ancestor. High substitution rates and biased nucleotide patterns have been the reason for the deviant tree topologies computed for individual sequences. A tree deduced from a concatenation of 205 protein sequences gave the same relationship as shown in Fig. 2 for E. coli, S. typhimurium, Y. pestis, and Buchnera (Lerat et al. 2003). In summary, these consistencies demonstrate that the above method of analyzing gene function and order generates a sound phylogeny, which is in most cases consistent with classical methods. As expected, the topology of the GO4genome net is less resolved for distantly related species (compare Fig. 2). Gene order conservation is lost rapidly when comparing species which are less related (Tamames 2001).

\section{Streptococci form Distinct Groups}

The genus Streptococcus is one of the most diverse and important human and agricultural pathogens. The genomes of streptococci exhibit extreme levels of evolutionary plasticity accompanied by a high level of gene gain and loss. It has been shown that recombination is an important 
Fig. 2 A neighbor net of $E$. coli, shigellae, and several other microbial species deduced from encoded gene function. GO4genome was used to compute a distance matrix. SplitsTree4 was utilized to generate and display a neighbor net. A local net-like structure indicates ambiguities. Thus, regions of unclear topology can be visualized. See "Materials and Methods" for species names

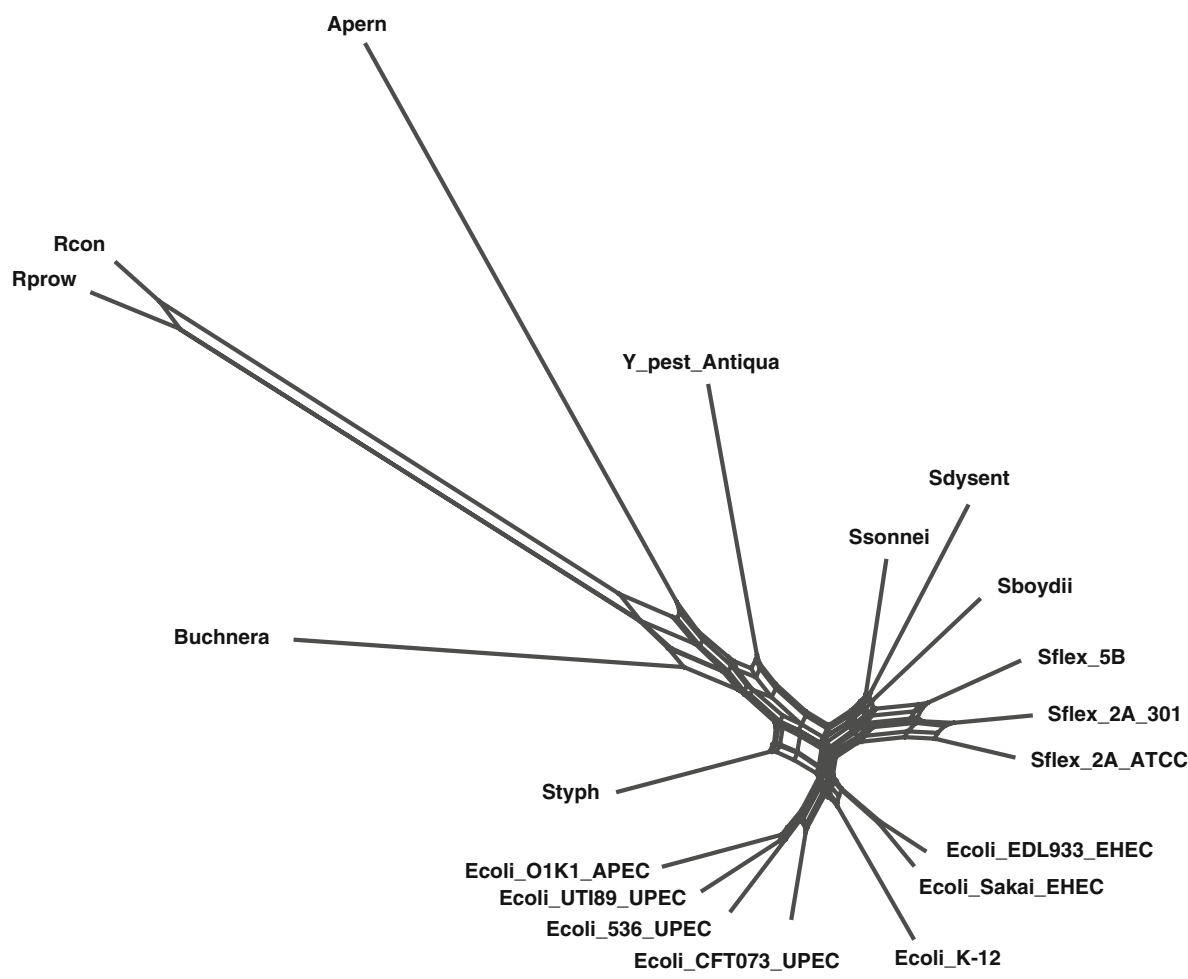

factor in the evolution of Streptococcus genomes (Lefébure and Stanhope 2007). Based on gene gain, loss, and duplication, core-based phylogenies have been determined for Streptococcus and, more specifically, for S. agalactiae and S. pyogenes strains (Lefébure and Stanhope 2007). According to this approach, S. pyogenes and S. agalactiae are closely related, as well as $S$. pneumoniae and $S$. suis. Additionally, a tree for Streptococcus has been deduced from a joint analysis of 504 single-copy genes (Anisimova et al. 2007). In this case, S. pyogenes and S. agalactiae have been most similar, as well as $S$. thermophilus and $S$. pneumoniae. Genome organization as deduced by GO4genome is in agreement with these findings and additionally identifies the genome structure of $S$. sanguinis as most similar to that of $S$. pneumoniae and $S$. suis; see Fig. 3. In addition, the net topology is concordant with findings deduced from an analysis of dnaJ and gyrB sequences (Itoh et al. 2006).

According to Lefébure and Stanhope (2007), among S. pyogenes strains the pairs (MGAS9429, MGAS2096; MGAS315, SSI-1) and (M1 GAS, MGAS5005) are most related. GO4genome predicted the same relationship; compare Fig. 3. However, for some species, like (MGAS8232, MGAS10394), the predictions differ. Additionally, the net indicates that the serovars M3 and M18 form one group, and M1, M2, M4, M12, and M28 a second group, which is less homogeneous. M5 and M6 lie isolated. In summary, the phylogenetic net showed a relatively low level of ambiguities. The analysis of genome organization clearly separated individual Streptococcus species and allowed the grouping of serovars. As can be seen, gene gain and loss had no major impact on the overall genome organization of the species.

Horizontal Gene Transfer Has Little Effect on the Genome Organization of Methanosarcina

So far, three genomes of Methanosarcina have been analyzed. The genomes differ significantly in size: the genome of M. mazei contains 3370 genes; that of M. barkeri, 3606 genes; and that of $M$. acetivorans, 4540 genes. It has been postulated that up to $30 \%$ of the $M$. mazei genes have been acquired via HGT (Deppenmeier et al. 2002). For $M$. mazei, $8.1 \%$ of its genes constitute larger genomic islands with atypical codon usage; for $M$. acetivorans this fraction is $10.8 \%$ (Merkl 2004). Thus, these genomes represent an appropriate set for testing the robustness of GO4genome against HGT and variations in genome size. We compiled a dataset consisting of the above Methanosarcina and Methanosaeta thermophila (a distantly related methanosarcinales), Methanospirillum hungatei, Methanoculleus marisnigri, Methanocorpusculum labreanum (three methanomicrobiales), three pyrococci, and two thermoplasmata. Figure 4 shows the resulting neighbor net. All species belonging to the same order were grouped in distinct subnets; the only exception was $M$. thermophila. It is known that the evolutionary relationship to Methanosarcina is a distant one: analysis of the 16S RNA gave the 
Fig. 3 A phylogenetic classification of streptococci based on encoded gene function and gene location. GO4genome was used to compute a distance matrix according to Formula (6). By means of SplitsTree4, a neighbor net was generated and plotted. Among $S$. pyogenes strains, several clusters are discernible

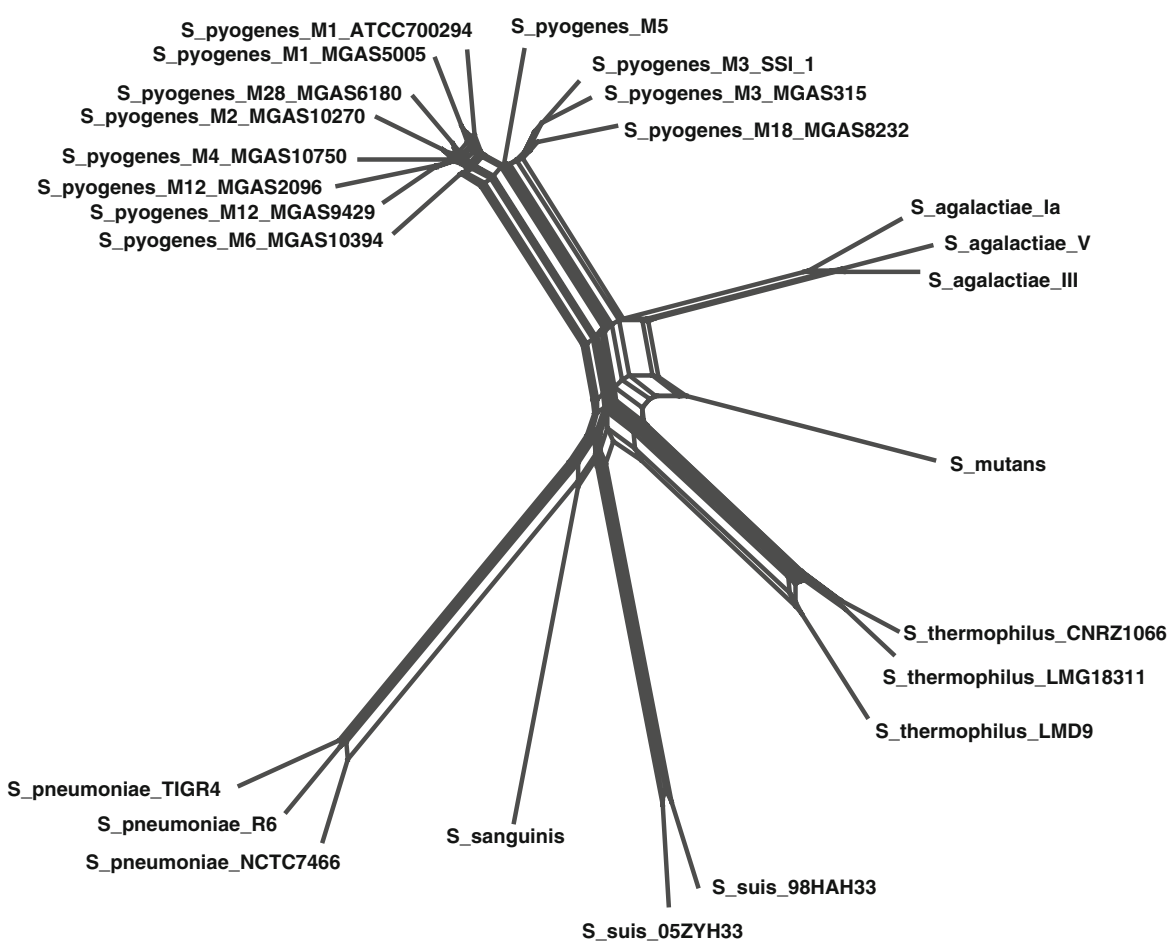

same local topology as shown in Fig. 4 for M. mazei, M. thermophila, and M. hungatei (Sekiguchi et al. 1998). Notably, the Methanosarcina species form a distinct subgroup, indicating that variations in genome size and larger

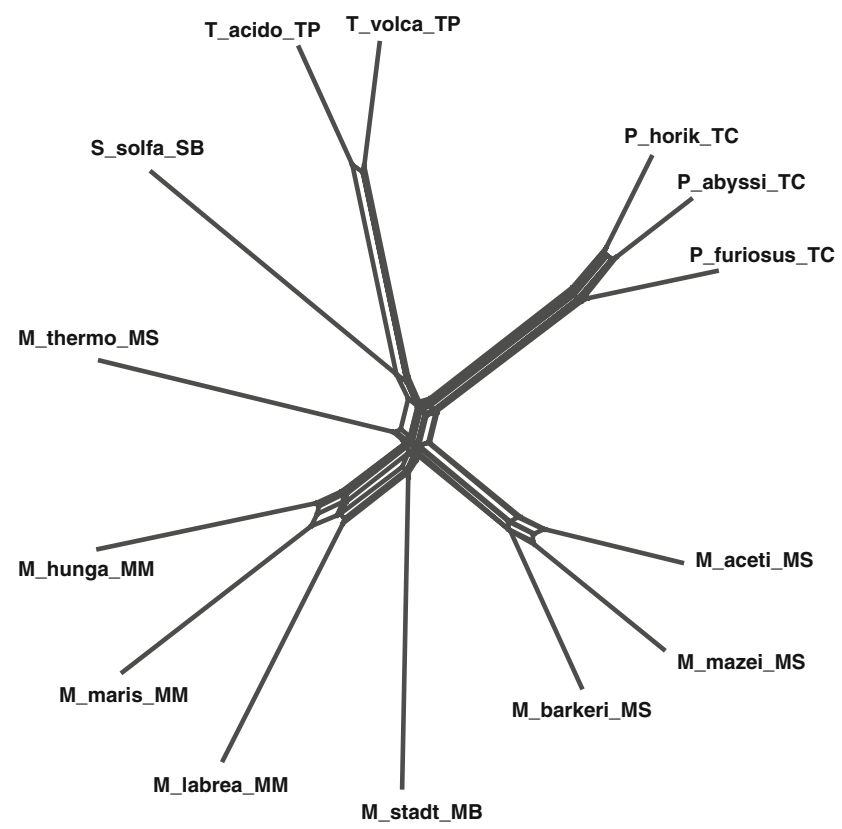

Fig. 4 A whole-genome phylogeny for methanosarcinales and other archaea. GO4genome was used to determine a distance matrix. SplitsTree4 was utilized for computation of a neighbor net and visualization. The suffixes indicate the lineage: $M S$ methanosarcinales, $M M$ methanomicrobiales, $T P$ thermoplasmatales, $T C$ thermococcales. See "Materials and Methods" for species names amounts of HGT have only a minor effect on the resolving power of GO4genome.

\section{GO4genome Groups Yersinia in a Novel Way}

Yersinia pestis is a Gram-negative bacterium and the causative agent of plague. $Y$. pestis is considered a recently emerged clone of $Y$. pseudotuberculosis, which evolved during the last 9000-40,000 years (Achtman et al. 2004). Originally, yersiniae were grouped into a "nonclassical" subspecies (containing Microtus) and three "classical" biovars, based on their ability to reduce nitrate and utilize glycerol: Antiqua (positive for both markers), Mediaevalis (do not reduce nitrate but utilize glycerol), and Orientalis (positive for nitrate reduction but do not utilize glycerol). Due to the latest analytical methods and molecular relatedness, $Y$. pestis strains were split into three major branches (Achtman et al. 2004; Auerbach et al. 2007). Branch 0 contains $Y$. pestoides isolates and the Microtus isolate 91001. 1.ORI subsumes bacteria related to Orientalis strains, classical Mediaevalis strains are referred to 2.MED, and Antiqua isolates are split into two distinct groups, 1.ANT and 2.ANT, which were isolated in Africa and East Asia, respectively. A MLVA analysis suggested that 2.MED and 2.ANT represent sister clades (Achtman et al. 2004). Based on the analysis of several parameters like SNPs and the genome-specific inactivation of genes, it has been postulated that the Antiqua and CO-92 strains belong to one branch, and KIM and Nepal516 to the second one. According to this analysis, 1.ANT is closely related to the 
Orientalis strain CO-92, while 2.ANT (represented by the Asian Antiqua strain Nepal516) is more closely related to the Mediaevalis strain KIM (Chain et al. 2006). Figure 5 shows that GO4genome proposed a different topology: one split separated $Y$. pestis Mediaevalis KIM5, Y. pestis biovar Microtus 91001, and Y. pestis Orientalis CO-92; a second one, the two Antiqua strains $Y$. pestis Antiqua and $Y$. pestis Nepal516; and a third, Y. pseudotuberculosis and Y. enterocolitica. This result indicates that the genome organization of biovars Mediaevalis (including Microtus) and Orientalis (represented by CO-92) is most similar; the same holds for the two representatives of the Antiqua biovar. For strain 91001, evolution from an ancient $Y$. pestis strain in a different lineage has been postulated (Song et al. 2004). According to GO4genome, its genome organization most resembles CO-92 and KIM5.

\section{Conclusions that can Be Drawn from Genome Organization}

The analyses introduced above exemplify the application of GO4genome and indicate the types of problems that can be studied. In the following, we summarize some results. The crenarchaeon Sulfolobus solfataricus and the euryarchaeon Thermoplasma acidophilum inhabit the same ecological niche. There is evidence for a large amount of HGT between these species (Ruepp et al. 2000); many genes are closely related (e.g., $\operatorname{trp} A$ and $\operatorname{trpB}$ [Merkl 2007]). However, Fig. 4 clearly indicates that the genome composition of these species is quite dissimilar. For M. mazei, $8.1 \%$ of its genes constitute larger genomic islands with atypical codon usage; for M. acetivorans this figure is $10.8 \%$ (Merkl
2004). Figure 4 shows, that despite these islands, their overall genome composition is still highly similar. Both findings suggest that HGT restructures genome content only locally.

Shigellae do not have a single evolutionary origin; however, many of their characteristics indicate convergent evolution (Pupo et al. 2000). Figure 2 makes clear that convergent evolution can be seen on the level of genome organization. More specifically, genome composition separates the three $S$. flexneri strains from $S$. boydii, S. sonnei, and $S$. dysenteriae, which constitute a separate cluster. In the case of $Y$. pestis, the similarity of genome organization proposes a convergent evolution of the Antiqua strains. The effect is detectable on the genome level; compare Fig. 5.

\section{Discussion}

What Is the Outcome of Classical Methods for the Cases Considered?

At first glance, it seems trivial to deduce the relationship of closely related prokaryotes. However, a comparison of the outcome of state-of the-art methods makes clear that this is not always a trivial task. Several cases are discussed below. The first example is the E. coli group. According to the analysis of tRNA genes (Withers et al. 2006) and 36 randomly chosen genomic regions (Elena et al. 2005), E. coli O157:H7 is a closer relative of E. coli $\mathrm{K}-12$ than S. flexneri. However, maximum likelihood analyses of core genomes and the ANI method identify S. flexneri as being more
Fig. 5 A whole-genome phylogeny for Yersinia strains. GO4genome was used to determine a distance matrix. SplitsTree4 was utilized for computation of a neighbor net and visualization. The net indicates that the genomes of the two Mediaevalis strains and of CO-92, as well as those of the two Antiqua strains, are most similar, respectively, when compared regarding gene function and their location

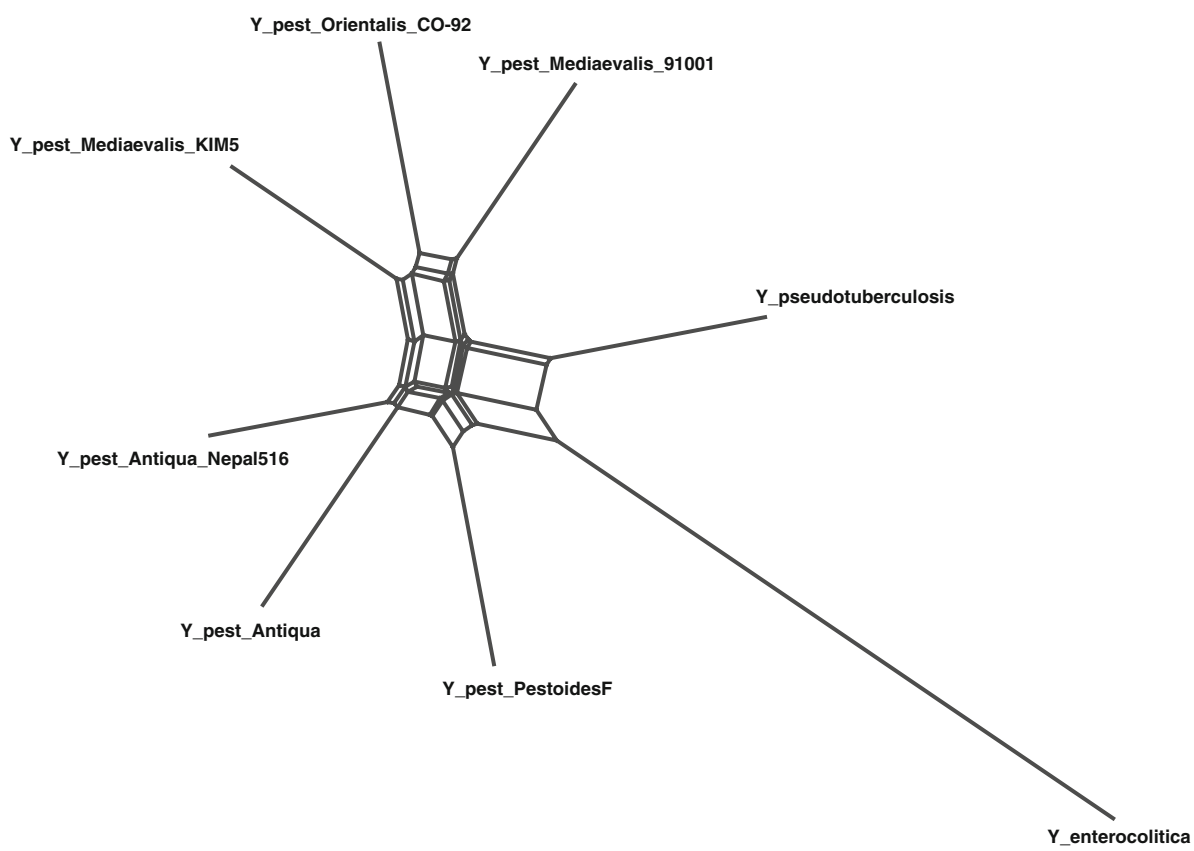


closely related to E. coli $\mathrm{K}-12$ than to $E$. coli $\mathrm{O} 157: \mathrm{H} 7$ (Konstantinidis et al. 2006). These differences might be due to the specific fate of individual genes. As has been pointed out, not all $\gamma$-proteobacterial core genes bear a similar phylogenetic signal supporting the same tree topology (Susko et al. 2006).

Shigellae have long been known to be closely related to E. coli. Due to biotyping, the genus has been divided into the four species $S$. boydii, $S$. dysenteriae, S. flexneri, and $S$. sonnei. Based on the analysis of eight housekeeping genes, it has been postulated that shigellae do not have a single evolutionary origin, which indicates convergent evolution of phenotypic properties (Pupo et al. 2000). An analysis of 23 housekeeping genes (Yang et al. 2007) has confirmed the clustering of shigellae into three main clusters, C1, C2, and $\mathrm{C} 3$. Clusters $\mathrm{C} 1$ and $\mathrm{C} 2$ consisted of $S$. dysenteriae and $S$. boydii strains; most of the strains (like F2a used here) constituting C3 were S. flexneri. The S. boydii strain Sb277 (used here) belonged to C1. The S. sonnei strain Ss046 (used here) was a direct neighbor of C1. The S. dysenteriae strain Sd197 (used here) laid isolated; the closest neighbors were E. coli EDL933 and E. coli Sakai. Contrariwise, an analysis of four chromosomal genes which were particularly polymorphic grouped Sd197 close to C1 and Ss046 close to C2 and C3 (Yang et al. 2007).

Based on the analysis of SNPs, a new nomenclature has been proposed for yersiniae (Achtman et al. 2004); see above. It has been postulated that Antiqua and CO-92 belong to one branch, and KIM and Nepal516 to a second one (Chain et al. 2006). A DNA microarray analysis of 22 strains of $Y$. pestis indicated that the two biovar strains Antiqua and Mediaevalis showed the most divergence from the CO-92 strain, and KIM and Nepal516 were clustered together (Hinchliffe et al. 2003). An analysis of CRISPR elements suggested that the Orientalis lineage branched out of the Antiqua strain earlier than the Mediaevalis biovar; the relative position of African Antiqua strains could not be fixed (Vergnaud et al. 2007). In summary, the above examples indicate that the phylogenetic signals studied highlight different aspects of genome evolution. This observation is in agreement with recent findings deduced from several methods of whole-genome phylogeny (McCann et al. 2008).

What Distinguishes Whole-Genome Analysis from Traditional Methods?

Several aspects of genome organization are not covered by classical methods. In many cases, bacteriophages are involved in the transfer of genomic islands. For S. flexneri 2a, 314 IS elements have been identified, which is more than sevenfold the content of E. coli K-12 (Jin et al. 2002).
A comparison of the $Y$. pseudotuberculosis genome with CO-92 and $\mathrm{KIM} 10+$ indicated that an extraordinary expansion of IS families has occurred since their divergence. It was deduced that the least common ancestor of CO-92 and KIM10+ carried 109 IS elements. Since their divergence from $Y$. pseudotuberculosis, KIM10+ and CO92 have undergone 10 or 18 rearrangements, respectively (Chain et al. 2004). Thus, it is quite likely that the insertion elements and/or the subsequent rearrangements they have generated played an important role in the speciation of $Y$. pestis strains (Chain et al. 2004). Y. pestis is actively undergoing reductive evolution and there is some evidence for convergent evolution (Chain et al. 2006).

In addition to the acquisition of novel genes by means of HGT, genetic rearrangements alter the position, the orientation, or the coding strand with respect to the origin of replication. As a consequence, gene dosage may be affected, as has been demonstrated for inversions in the genome of E. coli (Hill and Gray 1988). Depending on position, the effects of such rearrangements differ drastically (Esnault et al. 2007). Compared to E. coli sequences, 13 translocations and inversions of size $>5 \mathrm{~kb}$ have been identified in the genome of S. flexneri. It has been assumed that these rearrangements allow reoptimization of promoters in order to cope with selective pressure (Jin et al. 2002). The impact of rearrangements and their high frequency indicated above demand whole-genome analysis. In contrast to this approach, the analysis of a few genes or of SNPs covers a different aspect of phylogenomics, namely, the historical lineage of genes or genomes.

As has been shown, analysis of the common gene content has disadvantages as a measure for determination of phylogenies (Tamames 2001). In contrast, gene order conservation defines the course of evolution more precisely. In addition, its analysis does not depend on the presence of a certain set of genes. Along these lines, GO4genome supports a completely different aspect of "genome similarity," supplementing sequence-based methods and those elucidating the evolution of genes and genomes. As our approach assesses genomic signals which are influenced by more and different parameters than those related to the fate of single molecules, the grouping of species that differs from an analysis of classical markers is no surprise and does not judge the quality of any method. The networks resulting from GO4genome trace the evolutionary process of speciation based not on mutational events but on signal similarities in genome organization. As shown above for yersiniae, the genome organization of $Y$. pestis Antiqua and $Y$. pestis Antiqua Nepal516 is most similar; the same holds for KIM5, CO-92, and Mediaevalis 91001. Among Shigella, the genome organization of $S$. flexneri strains differs from that of $S$. boydii, $S$. $d y$ senteriae, and $S$. sonnei, which form a cluster. Most likely, 
effects which shape genomes above the gene level are responsible for these similarities.

As is the case for many other algorithms, we cannot prove the liability of our approach sensu strictu. However, the concordance of a great portion of the net topologies with well-established phylogenetic relations makes our findings highly plausible. We have demonstrated for several cases of inconsistencies that independent findings indicate them as well. In addition, it is unlikely that, just by chance, the genomes of (say) the Antiqua strains or of shigellae cluster in the pattern observed.

\section{Limitations and Further Improvements}

For prokaryotes, the organization of their genes in operons (Jacob and Monod 1961) and uberoperons (Lathe et al. 2000) is well established and it is known that the degree of genomic rearrangements increases constantly with the time of divergence (Suyama and Bork 2001). This holds even though there are discordant processes like HGT or varying rates of evolution or gene loss. However, these processes have been shown to add noise rather than a directional bias (Dutilh et al. 2004). In summary, these findings argue for analysis of genome organization. The above method is the first one utilizing the overall genome structure for determination of phylogenetic trees. So far, gene order has been exploited for gene pairs (Korbel et al. 2002) or rearrangements have been studied for a reduced set of genes in $\gamma$-proteobacterial genomes (Belda et al. 2005). The approach introduced with GO4genome eliminates some of the pitfalls of sequence-based phylogenies by comparing genes on function. For pairwise comparison of the genomes, it is not necessary to compare the respective sequences, which avoids false assignments. Due to the "fuzzy" scoring function, the selection of paralogues has only a minor effect on the identification of conserved genomic segments. As ontology is exploited, in situ replacements of genes maintaining the function of gene products have little impact on the phylogenetic distance. We believe that assessing HGT events in this way is at least a considerable alternative. Microbial genomes may contain a substantial number of duplicated genes, which argues for filtering (cf. Fig. 1). The above findings show that the proposed processing is appropriate to identify relevant gene series which can surrogate a cover.

Optimal applications for GO4genome are the study of serovars (see Fig. 3) or of closely related species (see Fig. 2).

Several improvements of GO4genome are conceivable. So far, the algorithm assesses gene function and location but not gene orientation. When comparing two genomes, the transcriptional orientation of each gene pair can be the same (positive polarity) or different (negative polarity).
However, how to integrate this signal into Formula (6) is unclear. The algorithm considers the length and size of rearrangements but not their location, e.g., with respect to the origin of replication. To do this, it would be necessary to model gene dosage for each species.

Above, we have focused on genomes consisting of a single chromosome. An analysis of several chromosomes is trivial; how to consider plasmids is unclear. Unfortunately, approaches exploiting gene order cannot be utilized for higher organisms: gene order is poorly conserved in eukaryotes (Huynen et al. 2001). The ultimate goal would be the comparison of all completely sequenced microbial genomes in order to compare genome organization. Due to the modular concept of our approach, such an analysis is feasible.

Acknowledgment The project was carried out within the BiotechGenoMik-Network Göttingen financed by the German Federal Ministry of Education and Research (BMBF).

\section{References}

Achtman M, Morelli G, Zhu P, Wirth T, Diehl I, Kusecek B, Vogler AJ, Wagner DM, Allender CJ, Easterday WR, Chenal-Francisque V, Worsham P, Thomson NR, Parkhill J, Lindler LE, Carniel E, Keim P (2004) Microevolution and history of the plague bacillus, Yersinia pestis. Proc Natl Acad Sci USA 101:17837-17842

Altschul SF, Gish W, Miller W, Myers EW, Lipman DJ (1990) Basic local alignment search tool. J Mol Biol 215:403-410

Anisimova M, Bielawski J, Dunn K, Yang Z (2007) Phylogenomic analysis of natural selection pressure in Streptococcus genomes. BMC Evol Biol 7:154

Auerbach RK, Tuanyok A, Probert WS, Kenefic L, Vogler AJ, Bruce DC, Munk C, Brettin TS, Eppinger M, Ravel J, Wagner DM, Keim P (2007) Yersinia pestis evolution on a small timescale: comparison of whole genome sequences from North America. PLoS ONE 2:e770

Bapteste E, Boucher Y, Leigh J, Doolittle WF (2004) Phylogenetic reconstruction and lateral gene transfer. Trends Microbiol 12: 406-411

Bapteste E, Susko E, Leigh J, Ruiz-Trillo I, Bucknam J, Doolittle WF (2008) Alternative methods for concatenation of core genes indicate a lack of resolution in deep nodes of the prokaryotic phylogeny. Mol Biol Evol 25:83-91

Belda E, Moya A, Silva FJ (2005) Genome rearrangement distances and gene order phylogeny in $\gamma$-proteobacteria. Mol Biol Evol 22:1456-1467

Chain PS, Carniel E, Larimer FW, Lamerdin J, Stoutland PO, Regala WM, Georgescu AM, Vergez LM, Land ML, Motin VL, Brubaker RR, Fowler J, Hinnebusch J, Marceau M, Medigue C, Simonet M, Chenal-Francisque V, Souza B, Dacheux D, Elliott JM, Derbise A, Hauser LJ, Garcia E (2004) Insights into the evolution of Yersinia pestis through whole-genome comparison with Yersinia pseudotuberculosis. Proc Natl Acad Sci USA 101:13826-13831

Chain PS, Hu P, Malfatti SA, Radnedge L, Larimer F, Vergez LM, Worsham P, Chu MC, Andersen GL (2006) Complete genome sequence of Yersinia pestis strains Antiqua and Nepal516: evidence of gene reduction in an emerging pathogen. J Bacteriol 188:4453-4463 
Ciccarelli FD, Doerks T, von Mering C, Creevey CJ, Snel B, Bork P (2006) Toward automatic reconstruction of a highly resolved tree of life. Science 311:1283-1287

Dalevi D, Eriksen N (2008) Expected gene-order distances and model selection in bacteria. Bioinformatics 24:1332-1338

Darling AC, Mau B, Blattner FR, Perna NT (2004) Mauve: multiple alignment of conserved genomic sequence with rearrangements. Genome Res 14:1394-1403

Daubin V, Gouy M, Perrière G (2002) A phylogenomic approach to bacterial phylogeny: evidence of a core of genes sharing a common history. Genome Res 12:1080-1090

Del Pozo A, Pazos F, Valencia A (2008) Defining functional distances over gene ontology. BMC Bioinformatics 9:50

Deppenmeier U, Johann A, Hartsch T, Merkl R, Schmitz RA, Martinez-Arias R, Henne A, Wiezer A, Bäumer S, Jacobi C, Brüggemann H, Lienard T, Christmann A, Bomeke M, Steckel S, Bhattacharyya A, Lykidis A, Overbeek R, Klenk HP, Gunsalus RP, Fritz H-J, Gottschalk G (2002) The genome of Methanosarcina mazei: evidence for lateral gene transfer between bacteria and archaea. J Mol Microbiol Biotechnol 4: 453-461

Dutilh BE, Huynen MA, Bruno WJ, Snel B (2004) The consistent phylogenetic signal in genome trees revealed by reducing the impact of noise. J Mol Evol 58:527-539

Elena SF, Whittam TS, Winkworth CL, Riley MA, Lenski RE (2005) Genomic divergence of Escherichia coli strains: evidence for horizontal transfer and variation in mutation rates. Int Microbiol $8: 271-278$

Esnault E, Valens M, Espéli O, Boccard F (2007) Chromosome structuring limits genome plasticity in Escherichia coli. PLoS Genet 3:e226

Fitz-Gibbon ST, House CH (1999) Whole genome-based phylogenetic analysis of free-living microorganisms. Nucleic Acids Res 27:4218-4222

Fukushima M, Kakinuma K, Kawaguchi R (2002) Phylogenetic analysis of Salmonella, Shigella, and Escherichia coli strains on the basis of the gyrB gene sequence. J Clin Microbiol 40:27792785

Hannenhalli S, Chappey C, Koonin EV, Pevzner PA (1995) Genome sequence comparison and scenarios for gene rearrangements: a test case. Genomics 30:299-311

Henz SR, Huson DH, Auch AF, Nieselt-Struwe K, Schuster SC (2005) Whole-genome prokaryotic phylogeny. Bioinformatics 21:2329-2335

Hill CW, Gray JA (1988) Effects of chromosomal inversion on cell fitness in Escherichia coli K-12. Genetics 119:771-778

Hinchliffe SJ, Isherwood KE, Stabler RA, Prentice MB, Rakin A, Nichols RA, Oyston PC, Hinds J, Titball RW, Wren BW (2003) Application of DNA microarrays to study the evolutionary genomics of Yersinia pestis and Yersinia pseudotuberculosis. Genome Res 13:2018-2029

Hughes D (2000) Evaluating genome dynamics: the constraints on rearrangements within bacterial genomes. Genome Biol 1:REVIEWS0006

Huson DH, Bryant D (2006) Application of phylogenetic networks in evolutionary studies. Mol Biol Evol 23:254-267

Huynen MA, Snel B, Bork P (2001) Inversions and the dynamics of eukaryotic gene order. Trends Genet 17:304-306

Itoh Y, Kawamura Y, Kasai H, Shah MM, Nhung PH, Yamada M, Sun X, Koyana T, Hayashi M, Ohkusu K, Ezaki T (2006) dnaJ and $\operatorname{gyr} B$ gene sequence relationship among species and strains of genus Streptococcus. Syst Appl Microbiol 29:368-374

Jacob F, Monod J (1961) Genetic regulatory mechanisms in the synthesis of proteins. J Mol Biol 3:318-356

Jin Q, Yuan Z, Xu J, Wang Y, Shen Y, Lu W, Wang J, Liu H, Yang J, Yang F, Zhang X, Zhang J, Yang G, Wu H, Qu D, Dong J, Sun
L, Xue Y, Zhao A, Gao Y, Zhu J, Kan B, Ding K, Chen S, Cheng H, Yao Z, He B, Chen R, Ma D, Qiang B, Wen Y, Hou Y, Yu J (2002) Genome sequence of Shigella flexneri 2a: insights into pathogenicity through comparison with genomes of Escherichia coli K12 and O157. Nucleic Acids Res 30:4432-4441

Johnson TJ, Kariyawasam S, Wannemuehler Y, Mangiamele P, Johnson SJ, Doetkott C, Skyberg JA, Lynne AM, Johnson JR, Nolan LK (2007) The genome sequence of avian pathogenic Escherichia coli strain O1:K1:H7 shares strong similarities with human extraintestinal pathogenic E. coli genomes. J Bacteriol 189:3228-3236

Konstantinidis KT, Ramette A, Tiedje JM (2006) Toward a more robust assessment of intraspecies diversity, using fewer genetic markers. Appl Environ Microbiol 72:7286-7293

Korbel JO, Snel B, Huynen MA, Bork P (2002) SHOT: a web server for the construction of genome phylogenies. Trends Genet $18: 158-162$

Kurland CG, Canback B, Berg OG (2003) Horizontal gene transfer: a critical view. Proc Natl Acad Sci USA 100:9658-9662

Kurtz S, Phillippy A, Delcher AL, Smoot M, Shumway M, Antonescu C, Salzberg SL (2004) Versatile and open software for comparing large genomes. Genome Biol 5:R12

Lathe WC 3rd, Snel B, Bork P (2000) Gene context conservation of a higher order than operons. Trends Biochem Sci 25:474-479

Lawrence JG, Ochman H (1997) Amelioration of bacterial genomes: rates of change and exchange. J Mol Evol 44:383-397

Lefébure T, Stanhope MJ (2007) Evolution of the core and pangenome of Streptococcus: positive selection, recombination, and genome composition. Genome Biol 8:R71

Lerat E, Daubin V, Moran NA (2003) From gene trees to organismal phylogeny in prokaryotes: the case of the $\gamma$-proteobacteria. PLoS Biol 1:E19

Lin GN, Cai Z, Lin G, Chakraborty S, Xu D (2009) ComPhy: prokaryotic composite distance phylogenies inferred from whole-genome gene sets. BMC Bioinformatics 10(Suppl 1):S5

Maddison DR, Swofford DL, Maddison WP (1997) NEXUS: an extensible file format for systematic information. Syst Biol 46:590-621

McCann A, Cotton JA, McInerney JO (2008) The tree of genomes: an empirical comparison of genome-phylogeny reconstruction methods. BMC Evol Biol 8:312

Merkl R (2004) SIGI: score-based identification of genomic islands. BMC Bioinformatics 5:22

Merkl R (2007) Modelling the evolution of the archeal tryptophan synthase. BMC Evol Biol 7:59

Nelson KE, Clayton RA, Gill SR et al (1999) Evidence for lateral gene transfer between archaea and bacteria from genome sequence of Thermotoga maritima. Nature 399:323-329

Ochman H, Lawrence JG, Groisman EA (2000) Lateral gene transfer and the nature of bacterial innovation. Nature 405: 299-304

Omelchenko MV, Makarova KS, Wolf YI, Rogozin IB, Koonin EV (2003) Evolution of mosaic operons by horizontal gene transfer and gene displacement in situ. Genome Biol 4:R55

Oshima K, Nishida H (2007) Phylogenetic relationships among mycoplasmas based on the whole genomic information. J Mol Evol 65:249-258

Pupo GM, Lan R, Reeves PR (2000) Multiple independent origins of Shigella clones of Escherichia coli and convergent evolution of many of their characteristics. Proc Natl Acad Sci USA 97:10567-10572

Rokas A, Williams BL, King N, Carroll SB (2003) Genome-scale approaches to resolving incongruence in molecular phylogenies. Nature 425:798-804

Rolland K, Lambert-Zechovsky N, Picard B, Denamur E (1998) Shigella and enteroinvasive Escherichia coli strains are derived 
from distinct ancestral strains of E. coli. Microbiology 144: 2667-2672

Ruepp A, Graml W, Santos-Martinez ML, Koretke KK, Volker C, Mewes HW, Frishman D, Stocker S, Lupas AN, Baumeister W (2000) The genome sequence of the thermoacidophilic scavenger Thermoplasma acidophilum. Nature 407:508-513

Saitou N, Nei M (1987) The neighbor-joining method: a new method for reconstructing phylogenetic trees. Mol Biol Evol 4:406-425

Sankoff D (1992) Edit distance for genome comparison based on nonlocal operations. In: Apostolico A, Crochemore M, Galil Z, Manber U (eds) Third annual symposium on combinatorial pattern matching, vol 644. Springer, Heidelberg, pp 121-135

Schlicker A, Domingues FS, Rahnenführer J, Lengauer T (2006) A new measure for functional similarity of gene products based on gene ontology. BMC Bioinformatics 7:302

Sekiguchi Y, Kamagata Y, Syutsubo K, Ohashi A, Harada H, Nakamura K (1998) Phylogenetic diversity of mesophilic and thermophilic granular sludges determined by $16 \mathrm{~S}$ rRNA gene analysis. Microbiology 144:2655-2665

Smith TF, Waterman MS (1981) Identification of common molecular subsequences. J Mol Biol 147:195-197

Snel B, Bork P, Huynen MA (1999) Genome phylogeny based on gene content. Nature Genet 21:108-110

Snel B, Bork P, Huynen MA (2002) Genomes in flux: the evolution of archaeal and proteobacterial gene content. Genome Res 12:17-25

Song Y, Tong Z, Wang J, Wang L, Guo Z, Han Y, Zhang J, Pei D, Zhou D, Qin H, Pang X, Han Y, Zhai J, Li M, Cui B, Qi Z, Jin L, Dai R, Chen F, Li S, Ye C, Du Z, Lin W, Wang J, Yu J, Yang H, Wang J, Huang P, Yang R (2004) Complete genome sequence of Yersinia pestis strain 91001, an isolate avirulent to humans. DNA Res 11:179-197
Susko E, Leigh J, Doolittle WF, Bapteste E (2006) Visualizing and assessing phylogenetic congruence of core gene sets: a case study of the $\gamma$-proteobacteria. Mol Biol Evol 23:1019-1030

Suyama M, Bork P (2001) Evolution of prokaryotic gene order: genome rearrangements in closely related species. Trends Genet 17:10-13

Swenson KM, Marron M, Earnest-DeYoung JV, Moret BME (2008) Approximating the true evolutionary distance between two genomes. ACM J Exp Algorithm 12:3.5

Tamames J (2001) Evolution of gene order conservation in prokaryotes. Genome Biol 2:RESEARCH0020

Tesler G (2002) GRIMM: genome rearrangements web server. Bioinformatics 18:492-493

Vergnaud G, Li Y, Gorgé O, Cui Y, Song Y, Zhou D, Grissa I, Dentovskaya SV, Platonov ME, Rakin A, Balakhonov SV, Neubauer H, Pourcel C, Anisimov AP, Yang R (2007) Analysis of the three Yersinia pestis CRISPR loci provides new tools for phylogenetic studies and possibly for the investigation of ancient DNA. Adv Exp Med Biol 603:327-338

Withers M, Wernisch L, dos Reis M (2006) Archaeology and evolution of transfer RNA genes in the Escherichia coli genome. RNA 12:933-942

Woese CR, Fox GE (1977) Phylogenetic structure of the prokaryotic domain: the primary kingdoms. Proc Natl Acad Sci USA 74: $5088-5090$

Xin C, Jie Z, Zheng F, Peng N, Yang Z, Stefano L, Tao J (2005) Assignment of orthologous genes via genome rearrangement. IEEE/ACM Trans Comput Biol Bioinform 2:302-315

Yang J, Nie H, Chen L, Zhang X, Yang F, Xu X, Zhu Y, Yu J, Jin Q (2007) Revisiting the molecular evolutionary history of Shigella spp. J Mol Evol 64:71-79 